\title{
Identification and profiling of growth- related microRNAs in Chinese perch (Siniperca chuatsi)
}

\author{
Jiagang Tu', Changxu Tian', Peiqi Zhao', Junxiao Sun ${ }^{1}$, Min Wang ${ }^{1}$, Qixue Fan ${ }^{1}$ and Yongchao Yuan ${ }^{1,2^{*}}$
}

\begin{abstract}
Background: MicroRNAs (miRNAs) are endogenous small non-coding RNAs that play important roles in the regulation of diverse biological processes in eukaryotes. Chinese perch (Siniperca chuatsi) is one of the most economically important fish species widely cultured in China. Growth is an extremely important characteristic in fish. Individual differences in body size are common in Siniperca chuatsi, which significantly influence the aquaculture production of Siniperca chuatsi. However, the underline growth-related regulatory factors, such as miRNAs, are still unknown.
\end{abstract}

Results: To investigate the growth-related miRNAs in Siniperca chuatsi, two RNA libraries from four growthrelated tissues (brain, pituitary, liver, and muscle) of Siniperca chuatsi at 6-month stage with relatively high or low growth rates (big-size group or small-size group) were obtained and sequenced using Solexa sequencing. A total of 252 known miRNAs and 12 novel miRNAs were identified. The expression patterns of these miRNAs in big-size group and small-size group were compared, and the results showed that 31 known and 5 novel miRNAs were differently expressed (DE). Furthermore, to verify the Solexa sequencing, five DE miRNAs were randomly selected and quantified by quantitative reverse transcription polymerase chain reaction (qRT-PCR). The results showed that their expression patterns were consistent with those of Solexa sequencing. In addition, Gene Ontology (GO) and Kyoto Encyclopedia of Genes and Genomes (KEGG) enrichment analysis of target genes of DE miRNAs was performed. It showed that the target genes were involved in multiple biological processes including metabolic process, suggesting that metabolic process played an important role in growth of fish.

Conclusions: Siniperca chuatsi is a popular and economically important species in aquaculture. In this study, miRNAs in Siniperca chuatsi with different growth rates were identified, and their expression profiles were compared. The data provides the first large-scale miRNA profiles related to growth of Siniperca chuatsi, which is expected to contribute to a better understanding of the role of miRNAs in regulating the biological processes of growth and possibly useful for Siniperca chuatsi breeding.

Keywords: MicroRNAs, Chinese perch, Growth, Deep sequencing, qRT-PCR

\footnotetext{
* Correspondence: yyc@mail.hzau.edu.cn

${ }^{1}$ College of Fisheries, Key Lab of Agricultural Animal Genetics, Breeding and Reproduction of Ministry of Education/Key Lab of Freshwater Animal Breeding, Ministry of Agriculture, Huazhong Agricultural University, Wuhan, Hubei 430070, China

${ }^{2}$ Freshwater Aquaculture Collaborative Innovation Center of Hubei Province, Wuhan, Hubei 430070, China
} 


\section{Background}

The Chinese perch (Siniperca chuatsi), with delicious flesh, is a popular and economically important fish species in many Asian countries. However, the cultured population of Siniperca chuatsi gradually exhibits growth depression, early sexual maturity, and disease susceptibility. Therefore, molecular techniques are needed to unveil these characteristics. Although the growth-related genes or single nucleotide polymorphisms of growth hormone gene of Siniperca chuatsi have been investigated $[1,2]$, the regulatory role of small RNAs, such as microRNA, on the growth of Siniperca chuatsi is still elusive.

MicroRNAs (miRNAs) are a class of endogenous, non-coding, 22-nt RNA molecules, which exist in a wide range of invertebrates and vertebrates [3]. It has been well known that miRNAs are involved in various biological processes in eukaryotes, including cellular proliferation, development, differentiation, metabolism, oncogenesis, and apoptosis [3-6]. They negatively regulate gene expression through binding to the 3' untranslated regions (UTRs), 5'UTRs, or the coding regions of target mRNA [7-9]. Since the first discovery of the miRNAs lin-4 and let-7 in Caenorhabditis elegans in 1993 [10], numerous miRNAs have been identified in mammals, plants, insects, worms, and viruses [11-14]. However, miRNA information is limited in fish species, with only 9 out of the 30,000 fish species present in miRBase [15-20].

High-throughput next-generation sequencing has become a powerful tool for transcriptomic and miRNA sequencing. MiRNAs have been identified in many fish species such as rainbow trout (Oncorhynchus mykiss) [21], Japanese flounder (Paralichthys olivaceus) [22], bighead carp (Hypophthalmichthys nobilis) [23], common carp (Cyprinus carpio) [24], and channel catfish (Ictalurus punctatus) [25]. However, the information on microRNA in Siniperca chuatsi is still unknown. Growth is one of the most important characteristics in fish. Growth-related miRNAs in blunt snout bream have previously been investigated through analyzing miRNA expression profiles from four growth-related tissues including brain, pituitary, liver, and muscle of big-size fish with relatively high growth rate and small-size fish with relatively low growth rate [26]. Individual differences in body size are common in Siniperca chuatsi, which significantly influence the aquaculture production of Siniperca chuatsi. In this study, we performed small RNA sequencing for the RNAs extracted from four growth-related tissues (brain, pituitary, liver, and muscle) of Siniperca chuatsi using individuals with relatively high and low growth rates (big-size group and small-size group). The miRNAs in the two groups of Siniperca chuatsi were identified and their expressions were compared. The $\mathrm{DE}$ miRNAs were further analyzed, and $\mathrm{GO}$ and KEGG enrichment analysis for the predicted target genes were performed. To our knowledge, our data provides the first survey of growth-related miRNAs in Siniperca chuatsi, which will contribute to a better understanding of the role of miRNAs in regulating the growth in fish.

\section{Methods}

\section{Animals and tissue collection}

All fish from the same family were fed with live mrigal Cirrhinus mrigala. All experimental procedures involving fish were approved by the institution of animal care and use committee of Huazhong Agricultural University. 60 fish with extreme phenotypes were selected at 3-, 6-, or 12-month stages, respectively: three small-size groups $\left(S_{3}, S_{6}\right.$ and $\left.S_{12}\right)$ and three big-size groups $\left(B_{3}, B_{6}\right.$ and $\left.B_{12}\right)$ from 1500 individuals [1]. Six fish in big-size or smallsize groups at 6-month stage were euthanized with tricaine methanesulfonate (MS-222) at $100 \mathrm{mg} / \mathrm{L}$. Four tissue samples including brain, pituitary, liver, and muscle of the fish were immediately frozen in liquid nitrogen upon surgical resection to avoid degradation and stored at $-80{ }^{\circ} \mathrm{C}$ prior for RNA isolation.

\section{Small RNA isolation and cDNA library construction}

Total RNAs were extracted from the tissue samples including brain, pituitary, liver, and muscle from bigsize group and small-size group of fish at 6-month stage using Trizol reagent (TaKaRa, Dalian, China) according to the manufacturer's protocol. RNA quality and quantity was measured using the NanoDrop 2000 (Thermo Scientific, Wilmington, DE, USA) and then standardized to $500 \mathrm{ng} / \mu \mathrm{l}$. Equal volumes of RNAs from different tissues of fish in the same group were combined into one pool. Small RNAs of $18-30 \mathrm{nt}$ in length were first isolated from the total RNAs by size fractionation, then were ligated with 3 '-RNA adapters,

Table 1 Primers for qRT-PCR used in this study

\begin{tabular}{lll}
\hline Name & Forward primers (5'-3') & Backward primers (5'-3') \\
\hline miR-10d-5p & TACCCTGTAGAACCGAATGTGTG & Universal primer ${ }^{a}$ \\
miR-126b-5p & CATTATTACTITTGGTACGCG & As above \\
miR-133a-3p & TTTGTCCCCTTCAACCAGCTG & As above \\
miR-142a-3p & TGTAGTGTTCCTACTTTATGGA & As above \\
miR-202-3p & AGAGGCATAGGGCATGGGAAAA & As above \\
U6 & CTCGCTTCGGCAGCACA & AACGCTTCACGATTTGCGT
\end{tabular}

${ }^{a}$ : It was provided from All-in-One ${ }^{T M}$ miRNA qRT-PCR Detection Kit 


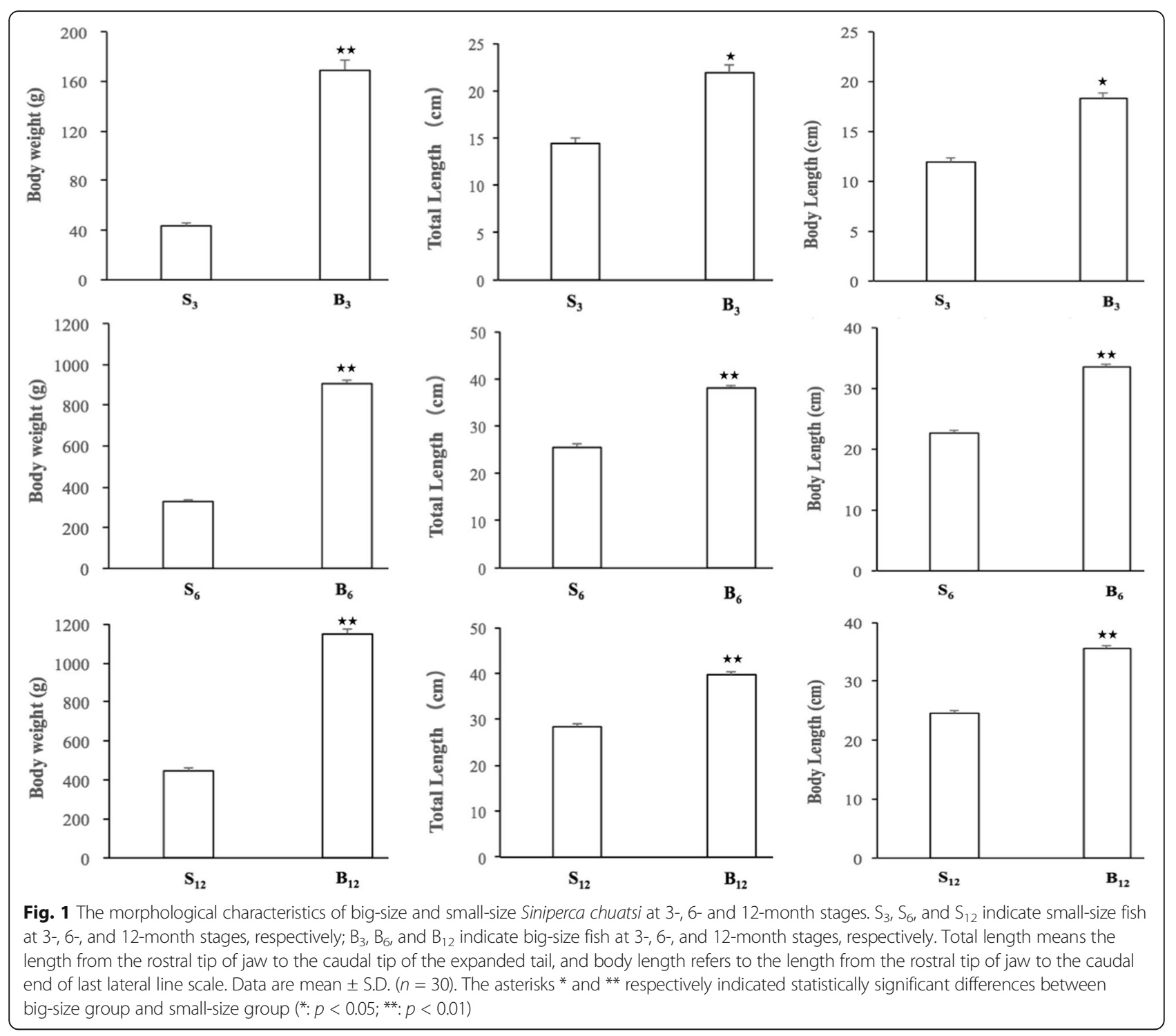

and finally created cDNA libraries using reverse transcription PCR. The amplified cDNA constructs were purified and sequenced by Illumina/Solexa technology (BMK, Beijing, China).

\section{Sequence data analysis}

The raw reads from Solexa sequencing were processed by evaluating sequencing quality. After eliminating low quality reads, adaptor sequences, and redundancy contaminant from raw reads, the clean reads were obtained. The clean reads with a length of 18-30 nt were blasted against the Rfam database and the GenBank noncoding RNA database to annotate rRNA, tRNA, snRNA, repeat associate small RNA, and other ncRNA sequences. Since the wholegenome sequence of Siniperca chuatsi is unknown, the remaining reads were used to map to the zebrafish (Danio rerio) genome. The mapped reads were then aligned with zebrafish miRNAs and the sequences with perfect match were identified as known miRNAs. The sequences that are not identical to the zebrafish miRNAs were used for novel miRNAs prediction using the Short Oligonucleotide Analysis Package (SOAP) software [27].

Table 2 Overview of the Solexa sequencing data

\begin{tabular}{|c|c|c|}
\hline & Small-size group & Big-size group \\
\hline Raw reads & $16,602,433$ & $16,048,981$ \\
\hline Clean reads & $15,351,486$ & $14,438,433$ \\
\hline Unannotated reads & $8,427,566$ & $7,962,496$ \\
\hline Mapped reads ${ }^{a}$ & $7,256,936$ & $7,156,763$ \\
\hline
\end{tabular}

a: After the rRNA, scRNA, snRNA, snoRNA, tRNA, and repbase were identified from the clean reads, the left unannotated reads were aligned with the genome of zebrafish to determine the mapped reads 


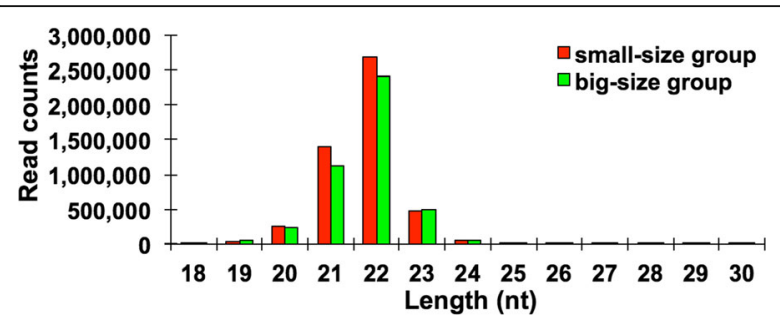

Fig. 2 Length distribution of small RNAs derived from Siniperca chuatsi. The small RNAs from small-size and big-size groups with length between 18 and 30 nt are analyzed

\section{Analysis of DE miRNAs}

To identify DE miRNAs between the big-size group and small-size group, the expression of miRNA in the two groups were normalized to obtain the expression of transcripts per million as described before [26]. The false discovery rate (FDR) $<0.05$ and $\left|\log _{2}{ }^{\text {(fold change) }}\right|>1$ was set as the threshold for significantly differential expression.

\section{qRT-PCR analysis of five DE miRNAs}

Five randomly selected miRNAs were detected by qRTPCR using the same RNA samples used for the construction of the small RNA libraries. The primers for the detection of these miRNAs were listed in Table 1. Quantitative PCR was performed using LightCycler ${ }^{\circ} 480$ Realtime PCR Instrument (Roche, Swiss) with $20 \mu \mathrm{l}$ PCR reaction mixture containing $2 \mu \mathrm{l}$ diluted cDNA, $400 \mathrm{nM}$ of each primer, and $10 \mu \mathrm{l}$ of qPCR Mix. Reactions were incubated in a 96-well optical plate (Roche, Swiss) at $95{ }^{\circ} \mathrm{C}$ for $10 \mathrm{~min}$, followed by 45 cycles of $95^{\circ} \mathrm{C}$ for $10 \mathrm{~s}$, $58{ }^{\circ} \mathrm{C}$ for $10 \mathrm{~s}$, and $72{ }^{\circ} \mathrm{C}$ for $10 \mathrm{~s}$, and ended with $95{ }^{\circ} \mathrm{C}$ at $5{ }^{\circ} \mathrm{C} / \mathrm{s}$ calefactive velocity to make the melt curve. All expression levels were normalized to the U6 gene [28]. Amplification results were analyzed using $2^{-\Delta \Delta C t}$ method, which achieve results for relative quantification. Ct represents the threshold cycle.

\section{Target gene prediction and analysis}

Considering that the genome information of Siniperca chuatsi is unavailable, we selected the zebrafish genome to predict the target genes with the strategy described previously [26]. GO and KEGG enrichment analysis were performed on the predicted target genes.

\section{Results and discussion}

\section{Morphological characteristics}

Individual differences in growth are common in fish but little is known about its genetic control [29]. In the present study, we found that the morphological characteristics including body weight, total length, and body length of big-size groups at 3-, 6-, and 12-month stages $\left(B_{3}, B_{6}\right.$, and $\left.B_{12}\right)$ were significantly higher than those of small-size groups $\left(S_{3}, S_{6}\right.$, and $\left.S_{12}\right)$, respectively (Fig. 1). Our previous study has revealed genes potentially influencing body size of Siniperca chuatsi at 6-month stage by transcriptome sequencing of mRNA libraries from big-size and small-size fish, and identified several upregulated growth factors (TGF-b1, TGF-b1r, PDGF and VEGFR2) and key genes (Ras, RasGFR, RasGRP, PKC and PI3K) in the PI3K-Akt and/or Ras-MAPK pathways [1]. However, the miRNAs involved in the growth of Siniperca chuatsi have never been investigated.

\section{Overview of Solexa sequencing data}

To identify the growth-related miRNAs in Siniperca chuatsi, two small RNA libraries were constructed from four growth-related tissue samples (brain, pituitary, liver, and muscle) of small-size group and big-size group of Siniperca chuatsi at 6-month stage. High-throughput Solexa sequencing was then performed on the small RNA libraries. A total of 16,602,433 and 16,048,981 raw reads were obtained from small-size group and big-size group, respectively (Table 2). After removal of the adapters, junk reads, and reads with length $<18 \mathrm{nt}$ or $>30 \mathrm{nt}, 15,351,486$
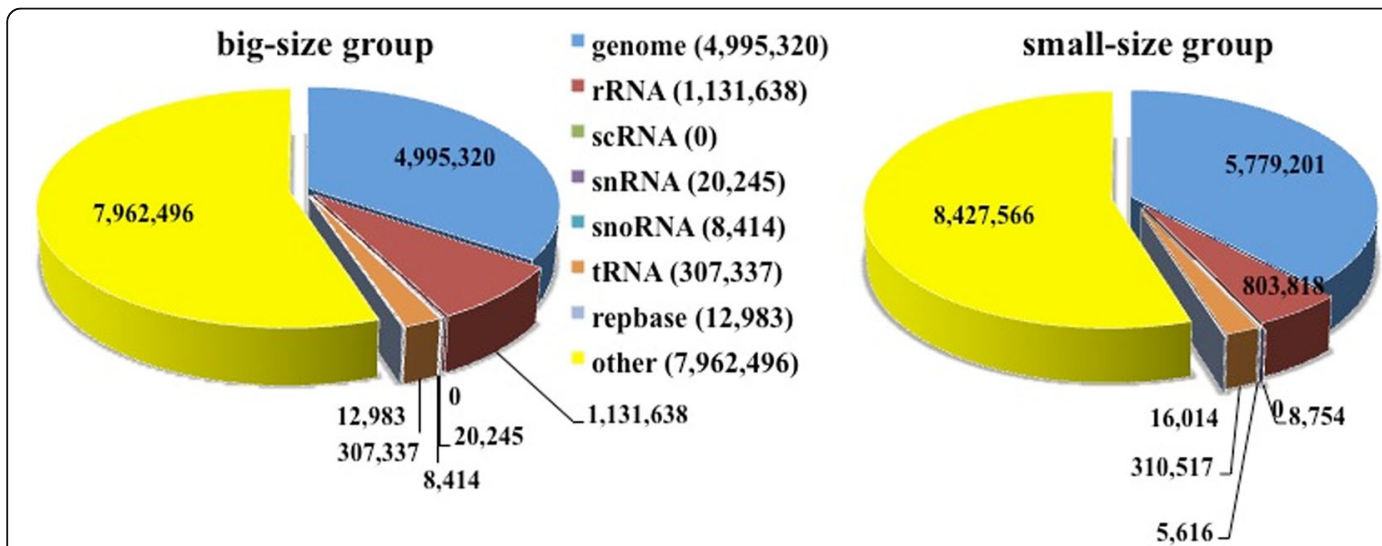

genome $(5,779,201)$

rRNA (803,818)

- scRNA (0)

- snRNA (8,754)

snoRNA $(5,616)$

- $\operatorname{tRNA}(\mathbf{3 1 0 , 5 1 7 )}$

repbase $(16,014)$

other $(8,427,566)$

5,616

Fig. 3 Annotation of small RNAs derived from Solexa sequencing of Siniperca chuatsi. The small RNAs from the big-size and small-size groups of Siniperca chuatsi were annotated after comparing with the NCBI GenBank and RFam database 
and 14,438,433 clean reads were obtained (Table 2). Length distributions of the clean reads revealed that the majority of the reads were about 21-23 nucleotides and the 22-nt reads were the most abundant (Fig. 2). After comparing the clean reads with the NCBI GenBank and Rfam database, the reads of rRNA, tRNA, scRNA, snRNA, snoRNA and repeat-associated small RNAs were annotated (Fig. 3). The remaining unannotated reads $(8,427,566$ for small-size group and 7,962,496 for big-size group, respectively) were tried to map with the genome of zebrafish. We found that $86.1 \%$ and $89.9 \%$ (7,256,936 and $7,156,763)$ of the unannotated reads from small-size group and big-size group were mapped to the genome of zebrafish, respectively (Table 2).

Table 3 Identification of DE miRNAs

\begin{tabular}{|c|c|c|c|c|c|}
\hline Name & Small-size group ${ }^{\#}$ & Big-size group & FDR & $\log _{2}^{\text {Fold changea* }}$ & Regulation \\
\hline miR-1 & $87,650.34892$ & $34,516.48053$ & 0 & -1.344474467 & down \\
\hline miR-10d-5p & 5761.977097 & $12,698.06025$ & 0 & 1.139972302 & up \\
\hline miR-126a-5p & 292.0125674 & 667.1046915 & 0 & 1.191882585 & up \\
\hline miR-126b-5p & 291.5991471 & 666.1259171 & 0 & 1.19180861 & up \\
\hline miR-133a-5p & 661.7481589 & 228.3340937 & 0 & -1.535136034 & down \\
\hline miR-133a-3p & 5668.268487 & 2069.548635 & 0 & -1.453591971 & down \\
\hline miR-133b-3p & 369.5977847 & 111.4404609 & 0 & -1.729682533 & down \\
\hline miR-137-5p & 1.5159 & 0.1398 & 0.001586 & -3.43873832 & down \\
\hline miR-137-3p & 42.99571545 & 19.43566383 & 0 & -1.1454833 & down \\
\hline miR-142a-5p & 494.1751141 & 1439.917022 & 0 & 1.54289143 & up \\
\hline miR-142a-3p & 403.7738662 & 943.8182075 & 0 & 1.224961334 & up \\
\hline miR-144-3p & 388.7529272 & 1023.798061 & 0 & 1.39700589 & up \\
\hline miR-150 & 0.2756 & 1.3982 & 0.007654 & 2.342922947 & up \\
\hline miR-153b-5p & 1.7915 & 0.6991 & 0.017438 & -1.357597303 & down \\
\hline miR-155 & 91.22808855 & 201.767359 & 0 & 1.14514292 & up \\
\hline miR-182-3p & 0.5512 & 2.0974 & 0.003435 & 1.927954234 & up \\
\hline miR-196a-5p & 162.6120007 & 25.30831045 & 0 & -2.6837512 & down \\
\hline miR-196b & 3.445169507 & 0.279649839 & 0.00000429 & -3.6231515 & down \\
\hline miR-19b-5p & 1.240261023 & 2.516848553 & 0.02231009 & 1.02090146 & up \\
\hline miR-200b-5p & 23.01373231 & 9.368269615 & 0 & -1.2966338 & down \\
\hline miR-202-5p & 2068.204159 & 485.8915957 & 0.000002 & -2.0896722 & down \\
\hline miR-202-3p & 20.2575967 & 2.516848553 & 0.000026 & -3.0088008 & down \\
\hline miR-203a-3p & 348.099927 & 132.4141989 & 0 & -1.3944436 & down \\
\hline miR-203b-5p & 4.547623749 & 1.118599357 & 0.000052 & -2.0234111 & down \\
\hline miR-203b-3p & 1.515874583 & 0.419474759 & 0.01697204 & -1.8534319 & down \\
\hline miR-205-5p & 75.65592238 & 19.85513859 & 0 & -1.929943 & down \\
\hline miR-206-3p & 8521.006645 & 2130.23265 & 0 & -2.0000128 & down \\
\hline miR-26a-2-3p & 0.689033901 & 2.516848553 & 0.00302765 & 1.86901469 & up \\
\hline miR-460-3p & 0.413420341 & 1.118599357 & 0.04857661 & 1.43608394 & up \\
\hline miR-499-5p & 252.1864079 & 125.4229529 & 0 & -1.0076885 & down \\
\hline miR-499-3p & 1.102454242 & 0.279649839 & 0.02966909 & -1.9793424 & down \\
\hline Novel-miR-1 & 1.3781 & 0.1398 & 0.00453429 & -3.3012443 & down \\
\hline Novel-miR-3 & 1.2403 & 0.4195 & 0.0357451 & -1.5639464 & down \\
\hline Novel-miR-8 & 1.5159 & 0.4195 & 0.01697204 & -1.8534319 & down \\
\hline Novel-miR-9 & 1.5159 & 0.5593 & 0.02860011 & -1.4384804 & down \\
\hline Novel-miR-11 & 1.1024 & 0 & 0.00339448 & -23.394275 & down \\
\hline
\end{tabular}

*: Fold change indicates the TPM values of each miRNA from big-size group were compared to those from small-size group

\#: The values from small-size group and big-size group indicate transcript per million (TPM) 


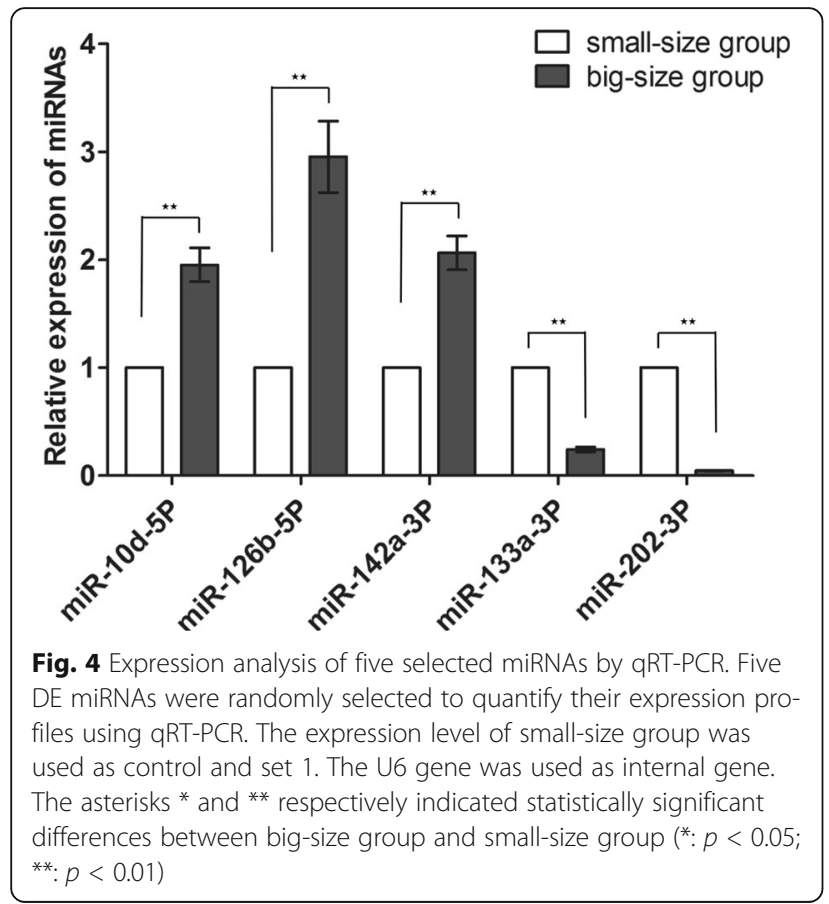

\section{Identification of known and prediction of novel miRNAs} in Siniperca chuatsi

Teleost is one of the most abundant species in vertebrate. However, only a total of 1250 miRNAs have been identified in 8 representative teleost species up to 2014 [15]. To identity the known miRNAs in Siniperca chuatsi, the clean reads were aligned with the zebrafish miRNAs in miRBase 20.0. A total of 252 known miRNAs were identified. Among them, 234 miRNAs were identified in both small-size group and big-size group. For the left 18 miRNAs, 9 miRNAs were expressed in small-size group, while the other 9 miRNAs were expressed in big-size group (Additional file 1). The expression levels of each miRNA in the two groups were calculated and presented as transcript per million (TPM) (Additional file 1). We found that the expression level of these miRNAs varied significantly, indicating that not only highly expressed but also lowly expressed miRNAs were detected by Solexa sequencing. Of the $252 \mathrm{miR}$ NAs, the most abundant three miRNAs were miR-1, miR-22a-3P, and miR-21, with the TPM value higher than 80,000 (Additional file 1).

The small RNAs that could not be aligned with the known zebrafish miRNAs were subjected to novel miRNA prediction. According to the criteria for miRNA prediction as previously [26], we finally obtained 12 novel miRNAs in Siniperca chuatsi. Among them, 11 miRNAs were found in both small-size group and bigsize group. Interestingly, the frequencies of these novel miRNAs were much lower than those of known miRNAs (Table 3 and Additional file 1).

\section{DE miRNAs}

The expression levels of the known and novel miRNAs in small-size group and big-size group were compared.

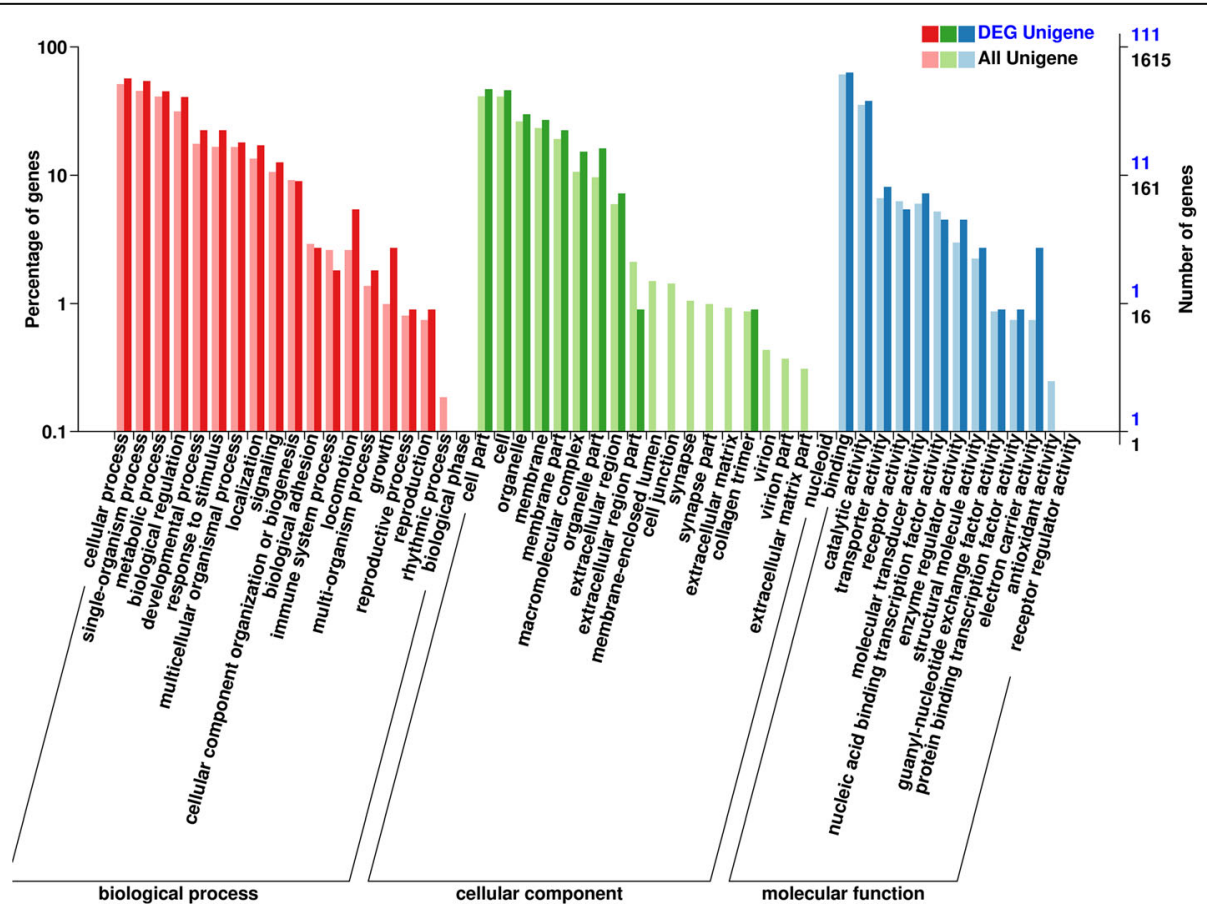

Fig. 5 GO enrichment analysis of target genes of all miRNAs as well as DE miRNAs between big-size group and small-size group 
Totally 31 known and 5 novel miRNAs were DE (FDR $<0.05$ and $\mid \log _{2}$ (fold change) $\mid>1$ ) when the expression level of the miRNAs in big-size group was compared to those in small-size group (Table 3). Of the 31 known miRNAs, 12 miRNAs were upregulated and 19 miRNAs were downregulated. These DE known miRNAs were then compared with previously reported potential growth-related miRNAs in other fish species. The DE miRNAs have been previously investigated in Portunus trituberculatus through comparing miRNA expression profiles between big-size fish and small-size fish. We found that the downregulation of miR-1 and upregulation of miR-150 were present in both Portunus trituberculatus and Siniperca chuatsi when the miRNAs in bigsize group were compared to those in small-size group [30]. These data suggested that the downregulation of miR-1 and upregulation of miR-150 might be involved in growth regulation in fish species. MiR-203 has been reported to inhibit cell growth through regulation of G1/ $\mathrm{S}$ transition by targeting Bmi-1 in myeloma cells [31].
Here, we found that three miR-203 isomiRs including miR-203a-3P, miR-203b-5P, and miR-203b-3P were downregulated (Table 3 ). Therefore, the downregulation of miR-203 might probably enhance the expression of Bim-1 and thus facilitate the cell growth in Siniperca chuatsi. In addition, miR-155 could promote cancer cell growth by repressing the tumor suppressor cyclin-D binding myb-like transcription fector 1 (DMTF1) [32]. In this study, we found that miR-155 was upregulated (Table 3), indicating that the upregulation of miR-155 might inhibit the expression of DMTF1 and thus promote the cell growth of Siniperca chuatsi. Although we identified several possible growth-related miRNAs here, it is still needed to be determined in the future.

In addition to the DE known miRNAs, we also identified 5 DE novel miRNAs from the 12 novel miRNAs, and all five novel miRNAs were downregulated (Table 3 ).

To validate the differential expression of the miRNAs between small-size group and big-size group, five randomly selected DE miRNAs including three upregulated

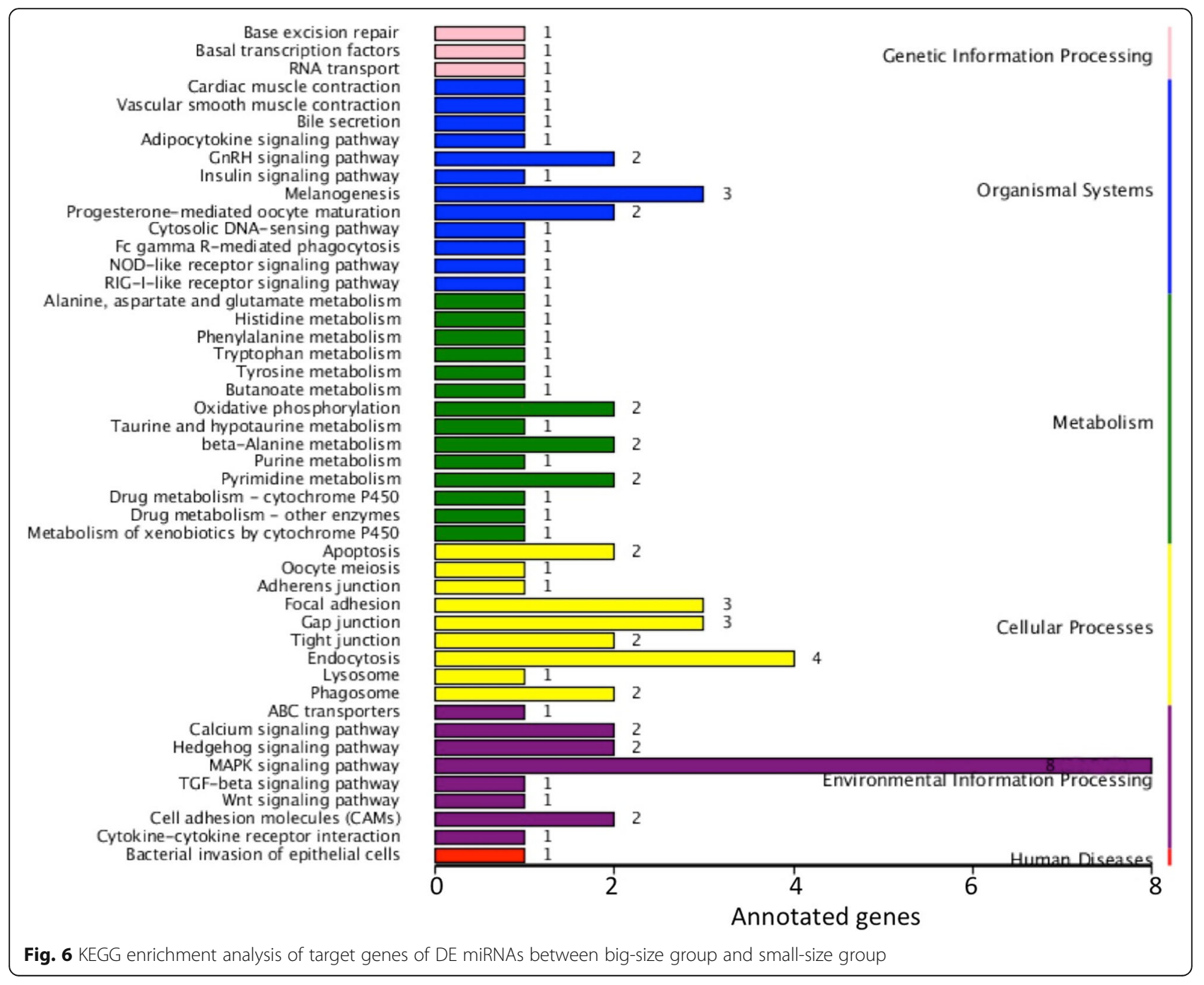


miRNAs (miR-10d-5P, miR-126b-5P, miR-142a-3P) and two downregulated miRNAs (miR-133a-3P and miR202-3P), were quantified using qRT-PCR. The mean normalized miRNA expression level was calculated and presented as the relative fold change. The results showed that all the five miRNAs exhibited a pattern consistent with the Solexa sequencing data (Fig. 4).

\section{Target gene prediction of DE miRNAs as well as GO and KEGG pathway enrichment}

Previous studies have revealed that the regulation of growth in fish is a complex regulatory process [29], and the potential growth-related genes have been investigated [33]. The analysis of target genes of DE miRNAs is important to illuminate the growth-related miRNAs. In this study, Miranda and RNAhybrid software were used to predict target genes according to zebrafish genome. A total of 172 target genes were identified for the $31 \mathrm{DE}$ miRNAs. Among the 172 target genes, 111 genes were annotated by GO database and 69 genes were annotated by KEGG database. The predicted target genes were classified and enriched via GO or KEGG analysis. GO enrichment analysis demonstrated that the target genes of DE miRNAs were divided into three categories: biological process, cellular component, and molecular function. In detail, these genes were involved in many biological processes, and the two most enriched categories were singleorganism process and metabolic process (Fig. 5). Interestingly, the GO enrichment analysis of differently expressed mRNAs between big-size group and small-size group of Siniperca chuatsi showed that the most enriched categories of biological processes was metabolic process. It indicated that metabolic process might play an important role in growth of Siniperca chuatsi.

KEGG analysis showed that enriched target genes were also involved in several metabolic processes (Fig. 6). In addition, we found that the most enriched categories of the target genes belonged to the Mitogen-activated protein kinase (MAPK) signaling pathway (Fig. 6). MAPK signaling pathway has been reported to be highly associated with the growth of cancer cells $[34,35]$. Therefore, whether the DE miRNAs observed in this study was associated with growth of Siniperca chuatsi via regulating the MAPK signal pathway is worthy of further study.

\section{Conclusion}

In summary, we identified 252 known miRNAs and 12 novel miRNAs from growth-related tissues (brain, pituitary, liver, and muscle) of Siniperca chuatsi using Solexa sequencing. Our study provides the first characterization of growth-related miRNAs in Siniperca chuatsi. Thirtyone known miRNAs showed differential expression between the big-size and small-size groups of Siniperca chuatsi. Functional annotation of the predicted target genes of the DE miRNAs revealed a broad range of the metabolic pathway and biosynthesis processes. These findings support the hypothesis that certain miRNAs might be essential in the regulation of growth, and it will be critical to develop new strategies for the molecular breeding of Siniperca chuatsi.

\section{Additional file}

Additional file 1: Target gene prediction and analysis of miRNAs. (XLS $1498 \mathrm{~kb}$ )

Abbreviations

DMTF1: Cyclin-D binding myb-like transcription fector 1; FDR: False discovery rate; GO: Gene Ontology; KEGG: Kyoto Encyclopedia of Genes and Genomes; MAPK: Mitogen-activated protein kinase; MiRNAs: MicroRNAs; qRTPCR: Quantitative reverse transcription polymerase chain reaction; SOAP: Short Oligonucleotide Analysis Package; TPM: Transcript per million; UTRS: Untranslated regions

\section{Acknowledgments}

The Fundamental Research Funds for the Central Universities (2013PY072, 2011PY030) and the National Natural Science Foundation of China

$(31,302,203,31,272,641)$ were used to purchase the fish, and other reagents, for RNA sequencing and data analysis.

\section{Funding}

This work was funded by the Fundamental Research Funds for the Central Universities (2013PY072, 2011PY030), and the National Natural Science Foundation of China $(31302203,31272641)$.

\section{Availability of data and material}

The data supporting the results of this article are included in the article and its additional files.

\section{Author's contributions}

TJG and YYC designed the experiments, TJG, TCX, ZPQ, SJX, and WM performed the experiments, FQX and TCX analyzed the data, TJG and YYC wrote the paper.

\section{Competing interests}

The authors declare that they have no competing interests

\section{Consent for publication}

Not applicable.

\section{Ethics approval and consent to participate}

The fish were obtained from Wuhan Fisheries Science Research Institute, Wuhan, Hubei, China. All experimental procedures involving fish were approved by the institution of animal care and use committee of Huazhong Agricultural University.

\section{Publisher's Note}

Springer Nature remains neutral with regard to jurisdictional claims in published maps and institutional affiliations.

Received: 26 December 2016 Accepted: 7 June 2017

Published online: 28 June 2017

\section{References}

1. Tian C, Li L, Liang XF, He S, Guo W, Lv L, et al. Identification of differentially expressed genes associated with differential body size in mandarin fish (Siniperca chuatsi). Genetica. 2016;144(4):445-55.

2. Tian C, Yang M, LV L, Yuan Y, Liang X, Guo W, et al. Single nucleotide polymorphisms in growth hormone gene and their association with growth traits in Siniperca chuatsi (Basilewsky). Int J Mol Sci. 2014;15(4):7029-36. 
3. Bartel DP. MicroRNAs: genomics, biogenesis, mechanism, and function. Cell. 2004;116(2):281-97.

4. Bushati N, Cohen SM. microRNA functions. Annu Rev Cell Dev Biol. 2007;23: 175-205.

5. Lu J, Qian J, Chen F, Tang X, Li C, Cardoso WV. Differential expression of components of the microRNA machinery during mouse organogenesis. Biochem Biophys Res Commun. 2005;334(2):319-23.

6. Rottiers V, Naar AM. MicroRNAs in metabolism and metabolic disorders. Nat Rev Mol Cell Biol. 2012;13(4):239-50

7. Zuo H, Yuan J, Chen Y, Li S, Su Z, Wei E, et al. A MicroRNA-mediated positive feedback regulatory loop of the NF-kappaB pathway in Litopenaeus vannamei. J Immunol. 2016;196(9):3842-53.

8. Jopling CL, Yi M, Lancaster AM, Lemon SM, Sarnow P. Modulation of hepatitis C virus RNA abundance by a liver-specific MicroRNA. Science. 2005: 309(5740):1577-81

9. Forman JJ, Coller HA. The code within the code: microRNAs target coding regions. Cell Cycle. 2010;9(8):1533-41.

10. Lee RC, Feinbaum RL, Ambros V. The C. elegans heterochronic gene lin-4 encodes small RNAs with antisense complementarity to lin-14. Cell. 1993; 75(5):843-54.

11. Li SC, Chan WC, Hu LY, Lai CH, Hsu CN, Lin WC. Identification of homologous microRNAs in 56 animal genomes. Genomics. 2010;96(1):1-9.

12. Yu XM, Zhou Q, Li SC, Luo QB, Cai YM, Lin WC, et al. The silkworm (Bombyx Mori) microRNAs and their expressions in multiple developmental stages. PLoS One. 2008;3(8):e2997.

13. Hafner M, Landgraf P, Ludwig J, Rice A, Ojo T, Lin C, et al. Identification of microRNAs and other small regulatory RNAs using CDNA library sequencing. Methods. 2008:44(1):3-12.

14. Wang LW, Liu HH, Li DT, Chen HB. Identification and characterization of maize microRNAs involved in the very early stage of seed germination. BMC Genomics. 2011;12(1):154.

15. Bizuayehu TT, Babiak I. MicroRNA in Teleost fish. Genome Biol Evol. 2014; 6(8):1911-37.

16. Bizuayehu TT, Lanes CF, Furmanek T, Karlsen BO, Fernandes JM, Johansen $S D$, et al. Differential expression patterns of conserved miRNAs and isomiRs during Atlantic halibut development. BMC Genomics. 2012;13:11.

17. Mennigen JA, Martyniuk CJ, Seiliez I, Panserat S, Skiba-Cassy S. Metabolic consequences of microRNA-122 inhibition in rainbow trout,Oncorhynchus mykiss. BMC Genomics. 2014;15:70.

18. Juanchich A, Bardou P, Rue O, Gabillard JC, Gaspin C, Bobe J, et al. Characterization of an extensive rainbow trout miRNA transcriptome by next generation sequencing. BMC Genomics. 2016;17:164

19. Hong XS, Qin JH, Chen R, Yuan LL, Zha JM, Wang ZJ. Identification and characterization of novel and conserved microRNAs in several tissues of the Chinese rare minnow (Gobiocypris rarus) based on illumina deep sequencing technology. BMC Genomics. 2016;17(1):283.

20. Xiao J, Zhong H, Zhou Y, Yu F, Gao Y, Luo Y, et al. Identification and characterization of microRNAs in ovary and testis of Nile tilapia (Oreochromis niloticus) by using solexa sequencing technology. PLoS One. 2014;9(1):e86821.

21. Ramachandra RK, Salem M, Gahr S, Rexroad CE 3rd, Yao J. Cloning and characterization of microRNAs from rainbow trout (Oncorhynchus mykiss): their expression during early embryonic development. BMC Dev Biol. 2008;8:41.

22. Fu Y, Shi Z, Wu M, Zhang J, Jia L, Chen X. Identification and differential expression of microRNAs during metamorphosis of the Japanese flounder (Paralichthys olivaceus). PLoS One. 2011;6(7):e22957.

23. Chi W, Tong C, Gan X, He S. Characterization and comparative profiling of MiRNA transcriptomes in bighead carp and silver carp. PLoS One. 2011;6(8): e23549.

24. Yan $X$, Ding L, Li Y, Zhang X, Liang Y, Sun $X$, et al. Identification and profiling of microRNAs from skeletal muscle of the common carp. PLoS One. 2012;7(1):e30925

25. Xu ZQ, Chen JP, Li XG, Ge JC, Pan JL, Xu XF. Identification and characterization of MicroRNAs in channel catfish (Ictalurus punctatus) by using Solexa sequencing technology. PLoS One. 2013;8(1):e54174.

26. Yi S, Gao ZX, Zhao H, Zeng C, Luo W, Chen B, et al. Identification and characterization of microRNAs involved in growth of blunt snout bream (Megalobrama amblycephala) by Solexa sequencing. BMC Genomics. 2013; 14:754.

27. Li R, Li Y, Kristiansen K, Wang J. SOAP: short oligonucleotide alignment program. Bioinformatics. 2008;24(5):713-4.
28. Zhu X, Li YL, Chen DX, Wu P, Yi T, Chen T, et al. Selection of reference genes for microRNA quantitative expression analysis in Chinese perch, Siniperca chuatsi. Int J Mol Sci. 2015;16(4):8310-23.

29. Shi Y, He MX. Differential gene expression identified by RNA-Seq and qPCR in two sizes of pearl oyster (Pinctada Fucata). Gene. 2014;538(2):313-22.

30. Ren X, Cui Y, Gao B, Liu P, Li J. Identification and profiling of growth-related microRNAs of the swimming crab Portunus Trituberculatus by using Solexa deep sequencing. Mar Genomics. 2016;28:113-20.

31. Wu SQ, Niu WY, Li YP, Huang HB, Zhan R. miR-203 inhibits cell growth and regulates $\mathrm{G} 1 / \mathrm{S}$ transition by targeting Bmi-1 in myeloma cells. Mol Med Rep. 2016;14(5):4795-801.

32. Peng $Y$, Dong $W$, Lin TX, Zhong GZ, Liao B, Wang B, et al. MicroRNA-155 promotes bladder cancer growth by repressing the tumor suppressor DMTF1. Oncotarget. 2015;6(18):16043-58.

33. Kocmarek AL, Ferguson MM, Danzmann RG. Differential gene expression in small and large rainbow trout derived from two seasonal spawning groups. BMC Genomics. 2014;15:57.

34. Wang Y, Hong X, Wang J, Yin Y, Zhang Y, Zhou Y, Piao HL, Liang Z, Zhang L, Li G et al: Inhibition of MAPK pathway is essential for suppressing RhebY35N driven tumor growth. Oncogene. 2016;36(6):756-65.

35. Cheng $S$, Zhang $X$, Huang N, Qiu Q, Jin Y, Jiang D. Down-regulation of S100A9 inhibits osteosarcoma cell growth through inactivating MAPK and NF-kappaB signaling pathways. BMC Cancer. 2016;16:253.

\section{Submit your next manuscript to BioMed Central and we will help you at every step:}

- We accept pre-submission inquiries

- Our selector tool helps you to find the most relevant journal

- We provide round the clock customer support

- Convenient online submission

- Thorough peer review

- Inclusion in PubMed and all major indexing services

- Maximum visibility for your research

Submit your manuscript at www.biomedcentral.com/submit
C Biomed Central 